\title{
Current Trends in Magnetic Resonance
}

\author{
Vladimir I. Chizhik ${ }^{1} \cdot$ Murat S. Tagirov $^{2}$
}

Published online: 4 January 2020

(c) Springer-Verlag GmbH Austria, part of Springer Nature 2020

This special issue of Applied Magnetic Resonance demonstrates a multeity of magnetic resonance and its applications. The problems of biology and medicine are of great importance in current science. One of the important achievements is magnetic resonance imaging (MRI) - an interdisciplinary field of science at the intersection of physics, chemistry, computer science, engineering, and medicine. Despite the numerous advantages of magnetic resonance methods, they have a significant drawback-low sensitivity that leads to a long acquisition time. This circumstance necessitates the search for new methodological techniques. Two articles in this special issue are devoted to magnetic resonance imaging and related questions.

A new approach to image acquisition is presented in the work of S. Shubin, V. Frolov, and K. Tyutyukin: "Display of phosphorus-containing components on lowfrequency magnetic resonance images using the method of double magnetic resonance". One of the promising fields in MRI diagnostics is the mapping of the distribution of nuclei other than ${ }^{1} \mathrm{H}$ because of the presence of such nuclei, in particular ${ }^{31} \mathrm{P}$, in molecules involved in important biological processes. The work presents the technique of displaying phosphorus-containing components via proton signals in the ultralow magnetic field (7 mT) using double magnetic resonance methods. A model substance in a phantom was trimethyl phosphate $\left(\mathrm{CH}_{3} \mathrm{O}\right)_{3} \mathrm{PO}$.

A distinctive feature of MRI is the possibility of changing the image contrast using NMR relaxation effects. The article "Investigations and Modeling the Effect of Magnetic Nanoparticles on MR Image Contrast" of Yu.V. Bogachev, Ya.Yu. Marchenko, A.V. Nikitina, and B.P. Nikolaevis is devoted to the investigations of NMR relaxation of protons in aqueous solution and $2 \%$ agar-agar gel in the presence of magnetic nanoparticles. To identify the effect of magnetic nanoparticles (MNP) on the contrast of magnetic resonance images, the dependences of the MR signal intensity on the parameters of the two pulse RF sequences (spin-echo, gradient-echo),

Vladimir I. Chizhik

v.chizhik@spbu.ru

Murat S. Tagirov

Murat.Tagirov@kpfu.ru

1 Saint Petersburg State University, Saint Petersburg, Russia

2 Kazan Federal University, Kazan, Russia 
most generally used in MRI, were simulated and analyzed for different values of the MNPs concentration. Recommendations for choosing the optimal values of pulse RF sequence parameters for MRI in the presence of MNPs are formulated. In addition, the prospects for the use of magnetic nanoparticles in MR-guided theranostics are discussed.

Another direction is presented by articles devoted to "solid-state" NMR. Currently, molecular ferroelectrics are of great interest because they have a number of significant advantages over well-known inorganic ferroelectrics due to their specific properties like environment-friendliness, lightweight, and flexibility. The modern trend in applied physics of ferroelectrics is the development of electronic devices with low-dimensional elements. One of such objects is investigated in the work "NMR studies of a nanocomposite based on molecular ferroelectric diisopropylammonium bromide" by N.I. Uskova, E.V. Charnaya, D.Yu. Podorozhkin, S.V. Baryshnikov, A.Yu. Milinskiy, I.V. Egorova, and D.Yu. Nefedov. The properties of these molecular ferroelectric particles embedded into a nanoporous opal matrix were studied by high-resolution ${ }^{13} \mathrm{C} \mathrm{NMR}$ in the temperature range from 295 to $450 \mathrm{~K}$. The main attention was focused on changes in temperatures of the ferroelectric phase transitions and on crystal structure evolution.

In last decades, much attention is paid to the development and study of solidstate thermoelectric converters (TEC), which have a number of advantages over traditional generators and refrigerators. Recently, it has been proposed to use magnetic semiconductors as effective thermoelectrics. One of the promising compounds is semiconductor mineral chalcopyrite $\mathrm{CuFeS}_{2}$. In the article, "NMR ${ }^{63,65} \mathrm{Cu}$ in a local field and relaxation of nuclear spins in $\mathrm{CuFeS}_{2}$ magnetic semiconductor" by S.O. Garkavyi, V.L. Matukhin, E.V. Schmidt, R.R. Khusnutdinov, and G.V. Mozzhukhin, the study of the spectral and relaxation parameters of NMR copper nuclei in a local field in natural samples of the semiconductor mineral chalcopyrite $\left(\mathrm{CuFeS}_{2}\right)$, which is characterized by a strong relationship of magnetic and electrical properties, is presented. The main attention was paid to the determination of the mechanism of nuclear spin-lattice relaxation.

The article "Isotope effect on diffusion in Nafion studied by NMR diffusometry" by A. Privalov, E. Galitskaya, V. Sinitsyn, and M. Vogel describes a very interesting and potentially important investigation of the proton mobility in Nafion 212, which is a hydrated perfluorosulfonic polymer used in fuel cells. The authors contributed to understanding of the molecular mechanism of diffusion processes. In particular, the isotope effect $\mathrm{H} \leftrightarrow \mathrm{D}$ on diffusion in proton-exchange membrane Nafion 212 was investigated using NMR diffusometry in a wide temperature range. Two diffusion mechanisms are discussed: (1) a hopping mechanism of hydrated hydronium ions ("vehicular" type-oxygen carriers of the protons) and (2) the Grotthuss mechanism, where the individual protons are transferred from one water molecule to another by breaking and forming hydrogen bonds.

Structural information in rigid and soft solids can be obtained by NMR spectroscopy via the measurements of dipole-dipole spin couplings, which are very sensitive to interatomic distances and their orientations. The work of T.P. Kulagina, G.E. Karnaukh, and I.Yu. Golubeva "Solid echo in three-spin groups with arbitrary constants of dipole-dipole interaction" develops a theoretical approach for solving problems 
of this type. The suggested approach allowed them to derive analytical expressions for modeling the shape of a solid-echo signal and its time evolution for an actual system of three-spin groups with arbitrary dipole-dipole interactions (DDI) in solids. Upon comparison with experiment, authors' results may help to estimate a ratio of DDI constants and elucidate the orientation of three-spin groups in a solid under study.

Further development of NMR spectroscopy is a matter of importance. Despite the fact that many routine programs have been developed for decoding NMR spectra, new tasks constantly arise that require an individual approach. One of such successful implementations is described in the article "NMR study of spatial structure and internal dynamic of adducts of ninhydrin-derived azomethineylide with cyclopropenes" by S.I. Selivanov, S. Wang, A.S. Filatov, and A.V. Stepakov. The 1,3-Dipolar cycloaddition of azomethineylides with alkenes is a universal synthetic tool for creating five-membered nitrogen-containing heterocyclic fragments, which are part of many natural alkaloids, as well as pharmacologically and biologically important compounds, and cyclopropenes occupy a special place among alkenes. Their unique reactivity due to high ring strain and low distortion energy makes them valuable synthetic intermediates. The authors synthesized a series of adducts of ninhydrinderived azomethineylide with cyclopropenes. The structure of the new products obtained was established and conformational mobility was also studied using NMR spectroscopy. In addition, a very rare phenomenon of scalar relaxation was found in these objects.

Multidimensional correlations are very powerful concepts in modern NMR spectroscopy for studies of molecular structures and dynamics. The work of E.I. Uskova, M.M. Doroginitskii, V.D. Skirda, and I.H. Fatkhutdinov "New approach to analyze of 2D-map $\mathrm{T}_{1}-\mathrm{T}_{2}$ " develops further 2D approaches in NMR. Based on the method of two-dimensional NMR-relaxometry, a technique was developed for determining the joint distribution of the correlation time and the Van Vleck second moments. The technique was used to analyze the characteristics of sorbed water in the system "clay rocks-argillite". Using the experimental results, the authors claim that the combination of traditional methods of testing structure properties with X-ray diffractometry and a new method of analyzing $2 \mathrm{D}$ relaxometry data will help to make recommendations for the development and use of inhibiting additives to drilling fluids for various types of clay-mudstone.

The phenomena of magnetic resonances provide the basis for different practical applications including the creation of quantum generators, amplifiers, and magnetometers. One of the basic devices of modern global navigation satellite systems (GPS, GLONASS, Galileo, etc.) is a rubidium atomic clock (quantum standard of frequency and time). E.N. Pestov, A.N. Besedina, D.E. Pestov, and V.V. Semenov discuss a problem of the frequency instability of atomic clocks in their article "On realization of extremely small shift frequency MR in a wide range of operating temperatures in rubidium atomic clocks". To solve the problem, the authors proposed a new type of the ${ }^{87} \mathrm{Rb}$ absorption cell with two dissimilar anti-relaxation components (wall coating + buffer gas, ${ }^{40} \mathrm{Ar}$ ) and created a special physical setup for optical spin pumping of ${ }^{87} \mathrm{Rb}$ atoms. The experimental data show the possibility of the realization of the daily frequency instability of $\sim 1 \cdot 10^{-14}$. 
We hope that the publications presented in this issue will attract attention of scientific community and promote cooperation between different scientific groups. We are grateful to all authors who have contributed to this special issue.

Publisher's Note Springer Nature remains neutral with regard to jurisdictional claims in published maps and institutional affiliations. 